\title{
Análise da atenção secundária em saúde bucal no estado de Minas Gerais, Brasil
}

\author{
Analysis of secondary care in oral health \\ in the State of Minas Gerais, Brazil
}

Patrícia Azevedo Lino ${ }^{1}$

Marcos Azeredo Furquim Werneck ${ }^{1}$

Simone Dutra Lucas ${ }^{1}$

Mauro Henrique Nogueira Guimarães de Abreu ${ }^{1}$

${ }^{1}$ Departamento de Odontologia Social e Preventiva, Faculdade de Odontologia, Universidade Federal de Minas Gerais. Av. Antônio Carlos 6627, Pampulha. 31.270-901 Belo Horizonte MG Brasil. maurohenriqueabreu@ gmail.com

\begin{abstract}
The scope of this study was to describe and analyze specialized outpatient procedures in periodontics, endodontics and oral surgery performed in the State of Minas Gerais in 2010. Secondary data for the procedures performed in healthcare facilities and registered in the Information System of the Unified Health System were used. Descriptive and bivariate analysis was performed, considering $p<0.05$. 707,559 procedures were performed in 2,547 health units distributed in 693 (81\%) counties in the State. Of these procedures, $55 \%$ are in the area of surgery, $28 \%$ in periodontics and $17 \%$ in endodontics. The units are predominantly public under municipal administration, $52.5 \%$ of which have an oral health team $(\mathrm{OHT})$ and $96.9 \%$ have no qualification as Dental Specialty Centers (DSCs). DSCs are more efficient than the other units $(p<0.001)$, but there is a significant percentage that fails to comply with the goals of the Ministry of Health. Municipalities that have DSCs have higher population, more OHTs, lower potential coverage and better HDI. A significant number of specialized procedures are performed in the State of Minas Gerais, especially in primary care settings. DSC establishments are more efficient than non-DSC, considering the average number of procedures. The minimum production goals are met by a minority of DSCs.
\end{abstract}

Keywords Secondary care, Health evaluation, Oral health
Resumo Este estudo descreveu e analisou os procedimentos especializados ambulatoriais nas áreas de periodontia, endodontia e cirurgia bucal em Minas Gerais, 2010. Utilizou-se dados secundários dos procedimentos realizados nos estabelecimentos de saúde e registrados no Sistema de Informação do Sistema Único de Saúde. A análise descritiva e bivariada foi realizada, considerando $p<0,05$. Foram executados 707.559 procedimentos em 2.547 unidades de saúde distribuídas em 693 (81\%) municípios. Destes procedimentos, $55 \%$ são da área de cirurgia, $28 \%$ da periodontia e $17 \%$ são da endodontia. As unidades são predominante públicas, sob administração municipal, $52,5 \%$ possuem alguma equipe de saúde bucal (ESB), 96,9\% não tem habilitação como Centros de Especialidades Odontológicas (CEO). Os $C E O$ são mais eficientes que as demais unidades ( $p<0,001$ ), mas há importante percentual que não cumpre as metas do Ministério da Saúde. Os municípios com CEO possuem maior população, mais ESB, menor cobertura potencial por estas e melhores IDH. Um significativo número de procedimentos especializados é realizado, especialmente em estabelecimentos de atenção básica. CEO são mais eficientes do que os não CEO, considerandose a média de procedimentos realizados. As metas de produção mínima são cumpridas por uma minoria de CEO.

Palavras-chave Atenção secundária, Avaliação em saúde, Saúde bucal 


\section{Introdução}

No Brasil, a atenção odontológica ficou caracterizada, durante anos, por atender grupos populacionais restritos, por meio de programas voltados para o curativismo, tendo como resultado uma baixa cobertura, tanto no aspecto assistencial como populacional ${ }^{1}$. A falta de acesso a serviços odontológicos não é um problema exclusivo do Brasil. Harris ${ }^{2}$, em um estudo no Reino Unido relatou que um pequeno, mas significativo, número de pessoas no sul de Cheshire apresentava problemas de acesso a cuidados odontológicos. Leake $^{3}$, em um estudo sobre a organização e prestação de serviços de odontologia, afirmaram que apesar dos cuidados de saúde serem um direito do cidadão canadense, graves desigualdades no acesso aos cuidados de saúde bucal ainda persistem. No Brasil, a atual Política Nacional de Saúde Bucal visa à reorganização da atenção primária especialmente por meio das Equipes de Saúde Bucal (ESB) na Estratégia de Saúde da Família (ESF) e Unidades Odontológicas Móveis. Visa também ampliação e qualificação da atenção especializada por meio dos Centros de Especialidades Odontológicas (CEO) e Laboratórios Regionais de Prótese Dentária (LRPD) e pela oferta de assistência odontológica na atenção terciária ${ }^{4,5}$.

Para um adequado funcionamento de um sistema de saúde é necessária a oferta de serviço em todos os níveis, sendo a atenção primária a principal porta de entrada no sistema. No Brasil, a prestação de serviços de saúde bucal apresentou sensível expansão dos níveis primário e secundário, com ênfase para o primeiro ${ }^{6}$. Em um sistema de saúde é necessário que haja uma eficiente interface entre atendimento odontológico primário e secundário ${ }^{6,7}$, ou seja, não basta apenas a existência dos níveis de atenção, mas também interlocução entre esses níveis.

A atenção secundária em odontologia no Sistema Único de Saúde pode ser realizada em diversas instituições públicas e privadas. Os CEO são estabelecimentos públicos de saúde, classificados como Clínica Especializada ou Ambulatório de Especialidade. São preparados para oferecer à população serviços de diagnóstico bucal, com ênfase em detectar câncer de boca, nos serviços de periodontia especializada, nos de cirurgia oral menor dos tecidos moles e duros e nos de endodontia e atendimento a portadores de necessidades especiais ${ }^{8}$.

Nos últimos anos, houve um avanço nos mecanismos e instrumentos de acompanhamento e avaliação utilizados pelas instancias gestoras do
Sistema Único de Saúde. Os sistemas de informação também diversificaram, disponibilizando um leque amplo de dados que tendem a ser mais integrados. Ainda que sob forte regulação federal, percebe-se uma busca pelo estabelecimento de instâncias de negociação entre os gestores, com um planejamento mais comunicativo ${ }^{9}$. Dentre os vários instrumentos de acompanhamento e avaliação em saúde pode-se citar, como exemplos, o Cadastro Nacional de Estabelecimentos de Saúde (CNES), os indicadores e parâmetros assistenciais de cobertura e produtividade e os manuais dos Sistemas de Informações Ambulatoriais e Hospitalares (SIA e SIH), dentre outros ${ }^{10}$.

Avaliar a atenção secundária em saúde bucal, prestada pelo SUS em Minas Gerais, até bem pouco tempo, seria difícil, visto que eram escassos os locais onde esse tipo de assistência à saúde existia. Após definições políticas que aumentaram o aporte de recursos e possibilitaram o crescimento dessa rede ${ }^{11}$, conhecê-la e analisá-la tornou-se um passo necessário. O Ministério da Saúde aponta metas mínimas de produtividade para os diversos tipos de CEO. A produção mínima necessária mensal para a área de periodontia é igual a 60,90 e 150 procedimentos por mês, respectivamente, para os CEO tipo I, II e III. Na área de endodontia, essa mesma produção é igual a 35, 60 e 95, para CEO tipo I, II e III, respectivamente. Finalmente, para os procedimentos de cirurgia, a produtividade mínima exigida é igual a 80,90 , 170 , respectivamente, para CEO tipo I, II e III $^{12}$. Assim, este estudo objetivou descrever e analisar os procedimentos odontológicos considerados de atenção secundária pela Política Nacional de Saúde Bucal no SUS no estado de Minas Gerais. Além disso, o estudo identificou e descreveu as unidades de saúde do tipo CEO e outras que realizam procedimentos odontológicos especializados no estado. Descreveu as características dos municípios onde estão implantadas. Comparou os municípios com habilitação para $\mathrm{CEO}$ com os demais que realizam procedimentos especializados sem essa habilitação, em relação à cobertura da atenção primária, procedimentos de atenção secundária e características do município. Finalmente, objetivou-se verificar o cumprimento da produção mínima estabelecida pelo Ministério da Saúde (MS) ${ }^{12}$ nos CEO.

\section{Métodos}

Para analisar a assistência secundária em saúde bucal em Minas Gerais foram utilizados os dados 
disponíveis nos sistemas de informação em saúde brasileiros $^{13}$. Minas Gerais localiza-se na região Sudeste e, de acordo com o censo de 2010, é o segundo estado brasileiro mais populoso, com 19.597.330 habitantes ${ }^{14}$.

Os dados de produção são armazenados nos Sistema de Informações Ambulatoriais do Sistema Único de Saúde (SIA/SUS) de onde foi feita sua coleta, empregando o banco de dados do Departamento de Informática do SUS (Datasus). As informações foram acessadas em dezembro de 2011 e a tabulação foi feita por meio do Programa Tab para Windows - Programa TABWIN do MS.

Todas as unidades de saúde que realizavam procedimentos considerados especializados nas áreas de endodontia, periodontia e cirurgia pela Política Nacional de Saúde Bucal ${ }^{12}$ foram incluídas na análise. Vale ressaltar que no grupo da cirurgia encontram-se os procedimentos de cirurgia oral menor como, por exemplo, aqueles associados à especialidade de estomatologia. Não foram analisados os procedimentos relacionados aos Laboratórios Regionais de Prótese Dentária, a ortodontia e a implantodontia, pois as políticas de incentivo aconteceram após um ano de análise da pesquisa. Os procedimentos para pacientes com necessidades especiais não foram analisados por serem, na maioria das vezes, da atenção primária, sendo difícil distinguir quais foram realizados fora dos Centros de Especialidades Odontológicas.

O primeiro passo foi levantar a produção de procedimentos especializados ambulatoriais em Minas Gerais segundo estabelecimento de saúde constante no Cadastro Nacional de Estabelecimentos de Saúde (CNES). O período de produção Datasus analisado foi de janeiro a dezembro de 2010. Com a lista de estabelecimentos que apresentaram produção no período, foram coletadas tanto as variáveis relacionadas a esses estabelecimentos como as dos municípios onde estão localizadas. Foram excluídas as unidades de saúde que não apresentaram qualquer uma destas variáveis (Figura 1). Dois bancos de dados foram construídos: um para as variáveis no nível das unidades de saúde e outro para as dos municípios.

As variáveis medidas no nível das unidades de saúde e municípios estão descritas na Figura 1.

Os bancos de dados foram exportados para o programa SPSS versão 19.0. A análise descritiva envolveu cálculo de proporções, medidas de tendência central e variabilidade. Para avaliação da normalidade das variáveis quantitativas nos dois bancos foi utilizado o teste de Kolmogorov
-Smirnov. Foi utilizado o teste de Mann-Whitney para comparar a produção dos procedimentos especializados entre as unidades classificadas ou não como CEO. O mesmo teste foi utilizado para comparar as variáveis população do município, número de ESB I (cirurgião-dentista e auxiliar de saúde bucal), ESB II (cirurgião-dentista, técnico de saúde bucal e auxiliar de saúde bucal), ESB I e II, cobertura potencial das ESB, IDH 1991, IDH 2000 entre municípios com e sem CEO. O nível de significância estatística adotado foi $\mathrm{p}<0,05$.

\section{Resultados}

\section{Características dos estabelecimentos de saúde}

Em 2010, em Minas Gerais, foram executados 707.559 procedimentos bucais especializados ambulatoriais, considerados de atenção secundária pela Política Nacional de Saúde Bucal. Esses procedimentos foram produzidos em 2.547 unidades de saúde distribuídas em 693 municípios, de um total de 853 municípios do estado. Foram realizados 388.955 procedimentos cirúrgicos, 118.836 endodônticos e 199.768 periodontais. Não houve distribuição normal para os procedimentos especializados avaliados $(\mathrm{p}<0,001)$.

Das 2.547 unidades de saúde que produziram algum tipo de procedimento especializado no ano de 2010, 1.779 (69,85\%) foram classificadas como Centro de Saúde/Unidade Básica de Saúde/ Posto de Saúde (Tabela 1).

Da classificação administrativa, 2.404 (94,4\%) estabelecimentos estão sob administração da esfera municipal, 118 (4,6\%) sob administração privada, $16(0,6 \%)$ sob administração estadual e $9(0,4 \%)$ sob administração federal.

Apenas 87 unidades $(3,4 \%)$ possuem alguma atividade de ensino no Cadastro Nacional de Estabelecimentos de Saúde. A maioria das unidades é da administração direta das Secretarias Municipais de Saúde, da Secretaria de Estado de Saúde ou do Ministério da Saúde ( $\mathrm{n}=2.381$ unidades, 93,5\%). Das 2.547 unidades estudadas, 1.336 (52,5\%) possuem alguma equipe de saúde bucal cadastrada, ou seja, são estabelecimentos que aderiram à Estratégia de Saúde da Família e prioritariamente deveriam oferecer à população procedimentos da atenção primária em saúde. A maioria das unidades de saúde $(\mathrm{n}=2469,96,9 \%)$ não é CEO, sendo 24 (0,9\%) CEO do tipo I, 53 $(2,1 \%)$ do tipo II, e apenas uma é do tipo III. Ou seja, dos 2.547 estabelecimentos, 78 possuem ha- 


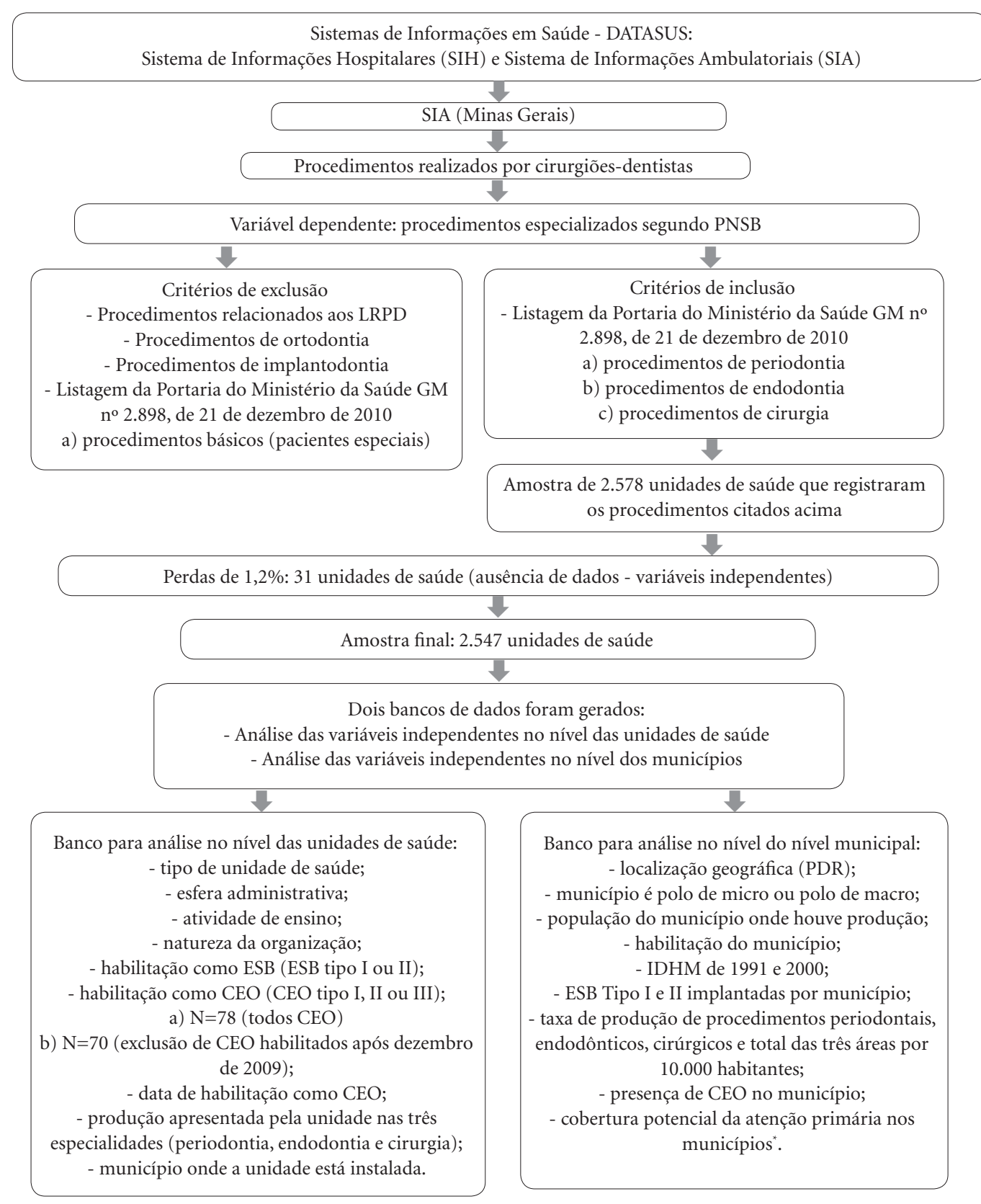

* O Departamento da Atenção Primária do MS adota um padrão nacional único para o cálculo da popualção coberta, que é o número médio estimado de 3.450 habitantes por equipe na ESF [16]. Esse parâmetro foi utilizado para o cálculo da cobertura potencial ${ }^{15,16}$. No numerador temos o número de equipes multiplicado por 3.450. O denominador é a população do município.

Figura 1. Processo de construção dos bancos de dados.

bilitação como CEO, sendo que 70 foram habilitados até dezembro de 2009 e oito após esse ano.

Houve diferenças entre a quantidade de procedimentos produzidos em cada uma das especialidades entre as unidades habilitadas como CEO e não CEO ( $p<0,001)$. Quando foram avaliados todos os estabelecimentos, inclusive os habilitados como CEO após dezembro de 2009 ( $\mathrm{n}=78$ ), a mediana de produção nas unidades de saúde que não possuem essa habilitação foi 16 procedimentos em cirurgia e zero em endodontia e periodontia. Para unidades que possuem habilitação como CEO, a mediana foi 925,50 procedimentos de cirurgia, 374,50 em endodon- 
Tabela 1. Distribuição dos tipos de unidades de saúde que realizaram procedimentos de atenção secundária em saúde bucal em Minas Gerais, 2010.

\begin{tabular}{lcc}
\hline Tipo de unidade de saúde & Frequência & \% \\
\hline $\begin{array}{l}\text { Centro de Apoio a Saúde da } \\
\text { Família (CASF) }\end{array}$ & 3 & 0,12 \\
Centro de Atenção Hemoterápica & 1 & 0,04 \\
e/ou Hematológica & & \\
Centro de Atenção Psicossocial & 1 & 0,04 \\
(CAPS) & & \\
Centro de Saúde/Unidade Básica & 1.779 & 69,85 \\
de Saúde /Posto de Saúde & & \\
Clinica Especializada/ & & \\
Ambulatório Especializado & 128 & 5,03 \\
Consultório & & \\
Hospital & 346 & 13,58 \\
Policlínica & 134 & 5,26 \\
Pronto Atendimento/ Pronto & 71 & 2,79 \\
Socorro Especializado/ Pronto & 43 & 1,69 \\
Socorro Geral & 1 & 0,04 \\
Secretaria de Saúde & & \\
Unidade de Serviço de Apoio de & 11 & 0,43 \\
Diagnose e Terapia & 11 & 0,43 \\
Unidade Mista & & \\
Unidade Móvel Terrestre & 18 & 0,71 \\
Total & 2.547 & 100 \\
\hline Fonte: DATSUS & &
\end{tabular}

Fonte: DATASUS tia e 721,50 em periodontia ( $\mathrm{p}<0,001$ ) (Tabela 2). Quando essa comparação é realizada com os estabelecimentos que foram habilitados para CEO até dezembro de $2009(n=70)$, observou-se que nas unidades que a possuem a mediana foi de 937,00 procedimentos de cirurgia, 380,00 em endodontia e 730,50 em periodontia $(p<0,001)$ (Tabela 2).

Em relação ao cumprimento das metas estabelecidas pelo Ministério da Saúde, a Tabela 3 cita a frequência relativa de estabelecimentos que não atingiram.

\section{Características dos municípios}

Com relação à população dos municípios mineiros, 50\% têm até 8.003 habitantes. Dos 853 municípios, 163 (19,1\%) não possuem nenhuma equipe de saúde bucal (ESB) implantada, 579 $(67,9 \%)$ possuem pelo menos uma do tipo I e $230(27 \%)$ pelo menos uma do tipo II.

A taxa de procedimentos especializados para cada 10.000 habitantes revelou que 160 municípios $(18,8 \%)$ apresentaram taxa igual a zero e $50,9 \%$ taxa de até 43 procedimentos para cada 10.000 habitantes (mediana 42). Com rela-

Tabela 2. Comparação entre produção de procedimentos odontológicos especializados entre os estabelecimentos classificados como CEO ou não, Minas Gerais, 2010.

\begin{tabular}{lrrr}
\hline Habilitação CEO & Procedimentos Cirúrgicos & Procedimentos Endodônticos & Procedimentos Periodontais \\
\hline NÃO & & & \\
$\mathrm{N}$ & 2.469 & 2.469 & 2.469 \\
Soma & 300.376 & 75.476 & 115.413 \\
Mediana & 16 & 0 & 0 \\
Mínimo & 0 & 0 & 0 \\
Máximo & 31.655 & 8.990 & 17.473 \\
SIM & & & \\
N & 78 & 78 & 78 \\
Soma & 88.579 & 43.360 & 84.355 \\
Mediana & 925,5 & 374,5 & 721,5 \\
Mínimo & 3 & 0 & 0 \\
Máximo & 6747 & 5.735 & 6215 \\
Valor p & $<, 001$ & $<0,001$ & $<0,001$ \\
SIM & & & 70 \\
N & 70 & 70 & 79.618 \\
Soma & 82.501 & 41.250 & 730,5 \\
Mediana & 937 & 380 & 0 \\
Mínimo & 3 & 0 & 6.215 \\
Máximo & 6.747 & 5.735 & $<0,001$ \\
Valor p & $<0,001$ & $<0,001$ & \\
& & &
\end{tabular}

*Valor p do teste de Mann-Whitney para comparações entre cada um dos procedimentos cirúrgicos, endodônticos e periodontais entre unidades não habilitadas como CEO e aquelas habilitadas até dezembro de $2009(\mathrm{~N}=70)$ e todas as unidades CEO em 2010 $(\mathrm{N}=78) ;{ }^{* *} \mathrm{CEO}$ habilitados até dezembro de 2009 comparados com unidades sem habilitação para CEO. 
ção à especialidade de cirurgia, 170 municípios (19,9\%) apresentaram taxa de procedimentos iguais a zero e $50,5 \%$ de até 26 procedimentos por 10.000 habitantes (mediana 26). Com relação à endodontia, 657 municípios $(77,0 \%)$ apresentaram taxa de procedimentos igual a zero para cada 10.000 habitantes durante o ano de $2010 . \mathrm{Na}$ periodontia, 646 municípios $(75,5 \%)$ apresentaram taxa de procedimentos igual a zero.

A frequência de municípios que apresentam CEO foi 71 , ou seja, apenas $8,3 \%$. Ainda existem microrregiões de saúde que não possuem nenhum CEO (33,8\%). As microrregiões com mais CEO são Belo Horizonte/Nova Lima/Caeté e Juiz de Fora/Lima Duarte/Bom Jardim de Minas. As macrorregiões Centro, Norte e Sul juntas concentram 50\% dos CEO existentes no ano de 2010. Dos municípios que possuem CEO, 51\% estão sob gestão municipal e $49 \%$ sob gestão dupla (Estado/Município), apenas 18,3\% dos mu- nicípios são polo de macrorregião e 63,4\% são polo de microrregião.

Existem diferenças estatisticamente significantes entre os resultados quando se comparam os valores encontrados dos municípios com e sem CEO. Aqueles que apresentam CEO possuem mais habitantes, mais ESB I e II, menor cobertura potencial e melhores IDH (1991 e 2000) quando comparados aos que não os possuem (Tabela 4).

\section{Discussão}

Há um considerável número de procedimentos odontológicos especializados realizados no estado de Minas Gerais. Tais procedimentos são executados, majoritariamente, em estabelecimentos públicos da atenção primária, sem atividade de ensino cadastrada. Os estabelecimentos cadastrados como CEO apresentaram maior eficiên-

Tabela 3. Frequência relativa de estabelecimentos habilitados como CEO que não atingiram as metas de produtividade estabelecidas pelo Ministério da Saúde, Minas Gerais, 2010.

\begin{tabular}{llccr}
\hline Tipo de CEO & $\begin{array}{c}\text { Procedimentos } \\
\text { Cirúrgicos }\end{array}$ & $\begin{array}{c}\text { Procedimentos } \\
\text { Endodônticos }\end{array}$ & $\begin{array}{c}\text { Procedimentos } \\
\text { Periodontais }\end{array}$ \\
\hline $\mathbf{N}=\mathbf{7 8}$ & Tipo I & $50,0 \%$ & $58,3 \%$ & $62,5 \%$ \\
& Tipo II & $62,3 \%$ & $84,9 \%$ & $62,3 \%$ \\
& Tipo III & $100,0 \%$ & $100,0 \%$ & $100,0 \%$ \\
$\mathbf{N}=\mathbf{7 0}$ & Tipo I & $47,8 \%$ & $56,5 \%$ & $60,9 \%$ \\
& Tipo II & $61,7 \%$ & $83,0 \%$ & $59,6 \%$ \\
\hline
\end{tabular}

Fonte: DATASUS

Tabela 4. Comparação entre características dos municípios que possuem CEO ou não, Minas Gerais, 2010.

\begin{tabular}{llrrrrrrr}
\hline CEO & & População & \multicolumn{1}{c}{ ESB } & ESB & ESB & Cobertura & IDH & IDH \\
\hline \multirow{4}{*}{ Não } & I & & II & I e II & potencial & $\mathbf{1 9 9 1}$ & $\mathbf{2 0 0 0}$ \\
& N & 782 & 782 & 782 & 782 & 782 & 782 & 782 \\
& Mediana & 7091 & 1 & 0 & 1 & 0,79 & 0,636 & 0,724 \\
& Mínimo & 815 & 0 & 0 & 0 & 0 & 0,42 & 0,57 \\
& Máximo & 2.375 .444 & 130 & 77 & 207 & 4,23 & 0,79 & 0,84 \\
& Soma & 12.113 .808 & 1.325 & 390 & 1715 & 604,01 & 490,57 & 558,87 \\
Sim & N & 71 & 71 & 71 & 71 & 71 & 71 & 71 \\
& Mediana & 57.361 & 3 & 0 & 5 & 0,39 & 0,701 & 0,777 \\
& Mínimo & 5.298 & 0 & 0 & 0 & 0 & 0,49 & 0,62 \\
& Máximo & 603.048 & 41 & 11 & 45 & 1,3 & 0,78 & 0,84 \\
& Soma & 7.481 .501 & 400 & 99 & 499 & 31,61 & 48,7 & 54,21 \\
& Valor de p $^{*}$ & $<0,001$ & $<0,001$ & 0,005 & $<0,001$ & $<0,001$ & $<0,001$ & $<0,001$ \\
\hline
\end{tabular}

* Teste de Mann-Whitney

Fonte: DATASUS 
cia, segundo conceito de Donabedian ${ }^{17}$, na execução de procedimentos especializados, apesar de não cumprirem a meta do governo brasileiro. A maioria dos municípios executa esses procedimentos, mas a taxa por habitantes é baixa. Poucos são os municípios que possuem CEO e esses apresentam maior população, maior número de ESB, menor cobertura da atenção primária em saúde bucal e melhores IDH se comparados aos municípios que não o possuem.

Tratando-se de avaliação em saúde, Donabedian ${ }^{17}$ propôs um modelo normativo de avaliação baseado em três componentes importantes do cuidado: estrutura, processo e resultado. No atual trabalho, avaliou-se estrutura (estabelecimentos de saúde) e processo (procedimentos executados).

A maioria dos procedimentos especializados é da área cirúrgica (55,0\%), seguido da periodontia $(28,2 \%)$ e, por último, os da endodontia $(16,8 \%)$. Um pouco mais que $50,0 \%$ dos municípios apresentaram taxa de até 43 procedimentos especializados para cada 10.000 habitantes e $18,8 \%$ dos municípios apresentaram taxa igual a zero nas três áreas. A situação das especialidades endodontia e periodontia é preocupante. Cerca de $77,0 \%$ dos municípios apresentaram taxa de procedimentos igual zero na especialidade de endodontia, e 75,5\% em periodontia. Abordando conceitos de acesso à saúde, Sanchez e Ciconelli ${ }^{18}$ citam que a incorporação de novas tecnologias na atenção especializada de países com cobertura universal aparece como alternativa interessante aos gestores de saúde. No caso da endodontia, a utilização de instrumentos rotatórios pode ser uma alternativa para agilizar a conclusão de tratamentos endodônticos ${ }^{19}$.

Mais de $80 \%$ dos municípios mineiros executaram algum procedimento especializado em saúde bucal no período de realização do estudo. Apesar de grande porcentagem de municípios mineiros terem realizado procedimentos especializados, afirmar que quase todos os municípios possuem cobertura para procedimentos especializados em saúde bucal seria um equívoco. Há uma dispersão na execução desses procedimentos em diversos estabelecimentos, mas não de forma eficiente $^{17}$, ou seja, a produção em unidades não classificadas como CEO é mais baixa.

Com relação às características dos estabelecimentos de saúde, a maioria dos procedimentos foi realizada em unidades básicas e que aderiram à estratégia de saúde da família, o que é contrário ao direcionamento atual da política nacional de saúde bucal ${ }^{4,5}$. Os estabelecimentos estão sob ad- ministração da esfera municipal em mais de $90 \%$ dos casos, o que significa que a política de descentralização/municipalização das ações e serviço públicos de saúde foi colocada em prática ${ }^{20}$.

Segundo Volpato e Scatena ${ }^{21}$ a criação do SUS visava romper com o modelo até então existente, de caráter curativo, discriminatório e mercantilista. Quando os resultados apontam que, no estado de Minas Gerais, apenas $16(0,6 \%)$ dos estabelecimentos estão sob administração privada, indica que este não exerce grandes influências na prestação de serviços especializados em saúde bucal no SUS. Diferente da odontologia, na área médica a participação do setor privado na atenção especializada do SUS é significante ${ }^{22}$. Nos últimos anos, na área médica, tem crescido a participação dos consórcios intermunicipais de saúde na prestação de serviços especializados ${ }^{23}$, fato não evidenciado na atenção secundária em odontologia em Minas Gerais.

As políticas atuais de saúde e educação deixam claro, em suas diretrizes, a importância do ensino em saúde estar próximo da realidade do SUS. Ressaltam a importância da academia formar profissionais de saúde que estejam preparados para lidar com a realidade da população $0^{24}$. O presente estudo demonstrou que apenas 3,4\% dos estabelecimentos possuíam alguma atividade de ensino cadastrada.

Apenas 3,1\% dos estabelecimentos possuem habilitação como CEO, sendo que a maioria é do tipo II. Metade dos municípios mineiros apresenta menos de 10.000 habitantes, o que significa que provavelmente poucos recursos financeiros de média e alta complexidade lhes são repassados. Assim, manter centros especializados pode se tornar inviável financeiramente. Além disso, a maioria dos profissionais especializados provavelmente concentra-se nos grandes centros urbanos. Figueiredo e Goes ${ }^{25}$ encontraram resultados semelhantes no estado de Pernambuco, quando avaliaram apenas as unidades com habilitação para CEO, a maioria também era tipo II.

A realidade de saúde pode apresentar diferentes características, ainda que em um mesmo município ${ }^{26}$. O presente estudo trabalhou os dados de estabelecimentos de saúde e de municípios, permitindo identificar diferenças regionais na atenção secundária em saúde bucal. Por se tratar de um estado com grande extensão territorial, o contraste entre as regiões de saúde é evidente. Observa-se a uma concentração de CEO em algumas regiões em contraste com os vazios assistenciais em outras. Segundo o Plano Diretor de Regionalização de Minas Gerais, o usuário do 
sistema deve encontrar serviços de atenção secundária dentro da microrregião em que reside ${ }^{27}$. O CEO deve ter abrangência microrregional com base populacional, sendo que o tipo II teria capacidade instalada para atender população referenciada de até 130.000 habitantes ${ }^{28}$. Os resultados demonstram que a maioria das microrregiões apresenta pelo menos um CEO. A grande área territorial, com realidades distintas entre municípios, pode também comprometer a referência intermunicipal. O tipo de instrumento de registro utilizado pelo SUS para esses procedimentos, não permitiu que fosse identificada a origem dos usuários atendidos no período do estudo. Uma mudança do tipo de instrumento de registro ambulatorial seria importante para monitoramento das pactuações intermunicipais, pois poderia favorecer a análise da eficiência dos CEO como abrangência microrregional.

Apesar da maioria dos procedimentos terem sidos realizados em unidades de saúde sem habilitação para $\mathrm{CEO}$, os resultados do estudo indicam que estes são mais eficientes na execução de procedimentos especializados em saúde bucal. Os resultados também sugerem que o tempo decorrido desde a implantação pode interferir na eficiência dos $\mathrm{CEO}$, ou seja, quando mais tempo em funcionamento, mais eficientes ${ }^{17}$. Mesmo apresentando um desempenho mais favorável, os resultados desse estudo apontaram que grande parte dos CEO não atingiu as metas do Ministério da Saúde para as áreas de cirurgia, periodontia e endodontia ${ }^{12}$. Berretta et al. ${ }^{29}$ propõem um modelo de avaliação da gestão municipal, e consideram que a eficácia da gestão é a habilidade do gestor em tomar decisões para o atendimento das metas estabelecidas pelas diretrizes para as ações em saúde no SUS. Nesse sentido, os resultados apontam para necessidade de revisão dessas me$\operatorname{tas}^{30} \mathrm{e} / \mathrm{ou}$ mudanças na gestão desses serviços.

Um ponto crítico, em relação às metas estabelecidas pelo Ministério da Saúde, seria o poder que cada serviço tem de direcionar a produção desses procedimentos no sentido de atingir essas metas. No caso de tratamento endodôntico, a meta deveria diferenciar os dentes a serem submetidos a tal tratamento, para que não se realize atendimentos de procedimentos menos complexos (dentes anteriores) em detrimento à maior necessidade da população (molares). Chaves e Silva ${ }^{31}$ citaram que, no caso da atenção secundária, um sistema de remuneração fixa pode desencorajar os profissionais a cumprirem metas mínimas de oferta de serviços. Problemas de desempenho e com o cumprimento de metas pelos
CEO já foram identificados em estudos anteriores $^{25,30}$. Uma alternativa para o estímulo ao cumprimento de metas poderia envolver prêmios de produtividade, reconhecendo o bom desempenho do profissional.

Não se deve pensar na atenção primária, sem a atenção secundária, pois as duas são essenciais para a integralidade da atenção em saúde bucal $^{7,26}$. É fundamental que a atenção primária à saúde seja ofertada em todos os municípios. Nestes, ela deve funcionar como porta de entrada do sistema de saúde, assumindo a função de organizar o cuidado integral. Além disso, sua presença fortalece o processo de descentralização para o nível municipal ${ }^{32}$. Fica evidente que a expansão da atenção primária em saúde bucal nos municípios mineiros, por meio da Estratégia de Saúde da Família, está bem consolidada. Mais de $80 \%$ dos municípios já possuem pelo menos uma equipe de saúde bucal. Diante da necessidade de que as ações de saúde bucal sejam organizadas em rede ${ }^{33}$, ter uma cobertura adequada da atenção primária favorece o sistema de referência e contrarreferência entre atenção primária e secundária. A política de organização da atenção secundária nos CEO parece ser adequada. Esses estabelecimentos estão situados em municípios polo. No entanto, a menor cobertura da atenção primária nestes polos é um aspecto que gera preocupação, uma vez que nestes municípios apresenta menor cobertura.

Os municípios que prestam atendimento especializado por meio dos CEO possuem, em números absolutos, maior número de ESB implantadas. Mas quando é feita a correção pela população do município, ou seja, calculada a cobertura potencial, em geral possuem menor cobertura da atenção primária em saúde bucal. Possuem também melhor Î́ndice de Desenvolvimento Humano quando comparado aos municípios sem CEO. Para estes, na atenção primária, houve direcionamento específico dos recursos de acordo com as diferentes necessidades da população, reveladas pela utilização de indicadores sociais $^{26}$. Os resultados desse trabalho indicam que, no caso da atenção secundária, o direcionamento dos recursos foi para municípios que apresentam melhores indicadores sociais.

Se, por um lado, a utilização de dados secundários dos sistemas de informação em saúde pode ser considerada uma limitação deste estudo $^{25,34}$, em termos de gestão, a facilidade de acesso às informações desses sistemas ajuda na apropriação de informações por parte do gestor, possibilitando a avaliação das políticas de saúde 
adotadas $^{21,25,31,35}$. Segundo Tanaka e Tamaki ${ }^{36}$, a incorporação da avaliação por aqueles que têm o poder de decisão e de implementar ações, é condição essencial para institucionalizar a avaliação na gestão dos serviços em saúde. A gestão é uma prática administrativa complexa. Nesse processo, o gestor utiliza de conhecimentos, técnicas e procedimentos que lhe permitam conduzir o funcionamento dos serviços de saúde na direção dos objetivos definidos. Esforços por parte do Ministério da Saúde brasileiro para estímular a apropriação do conhecimento científico na gestão da saúde já acontecem. Como exemplo podemos citar o projeto Pesquisa Saúde, que tem como objetivo aproximar dos gestores o conhecimento científico produzido ${ }^{37}$. A utilização dos dados dos serviços de saúde é relevante para avaliação da atenção secundária em saúde bucal no
Sistema Único de Saúde, bem como de outros sistemas de saúde.

\section{Conclusão}

A quantidade de procedimentos especializados realizados no estado de Minas Gerais foi significativa. A maioria dos procedimentos de atenção especializada é realizada em estabelecimentos de atenção básica. Estabelecimentos CEO são mais eficientes do que os não $\mathrm{CEO}$, considerando-se a média de procedimentos realizados. Os municípios que apresentam CEO possuem maior população e melhor IDH, mais ESB I e II, mas menor cobertura potencial quando comparados aos que não possuem CEO. As metas de produção mínima são cumpridas por uma minoria de CEO.

\section{Colaboradores}

PA Lino foi responsável pela coleta, análise de dados e redação do artigo. MAF Werneck contribuiu substancialmente para a construção do referencial teórico e na leitura crítica do artigo científico. SD Lucas e MHNG Abreu foram orientadores do trabalho, contribuindo com a construção do referencial teórico-metodológico, interpretação dos resultados e redação do artigo. Todos os autores revisaram e aprovaram a versão final do artigo.

\section{Referências}

1. Oliveira JLC, Saliba NA. Atenção odontológica no Programa de Saúde da Família de Campos dos Goytacazes. Cien Saude Colet 2005; 10(Supl.1):297-302.

2. Harris R. Access to NHS dentistry in South Cheshire: a follow up of people using telephone helplines to obtain NHS dental care. Br Dent J 2003; 195(8):457-461.

3. Leake JL. Why Do We Need an Oral Health Care Policy in Canada? J Can Dent Assoc 2006; 72(4):317-317j.

4. Souza TMS, Roncalli AG. Saúde bucal no Programa de Saúde da Família: uma avaliação do modelo assistencial. Cad Saude Publica 2007; 23(11):2727-2739.

5. Brasil. Ministério da Saúde (MS). Portal da Saúde. Brasil Sorridente. Brasília. [acessado 2014 jun 14]. Disponível em: http://portal.saude.gov.br/portal/saude/area. cfm?id_area $=406$

6. Brasil. Ministério da Saúde (MS). Secretaria de Atenção à Saúde, Departamento de Atenção Básica, Coordenação Nacional de Saúde Bucal. Diretrizes da política nacional de saúde bucal. Brasília, 2004. [acessado 2014 jun 14]. Disponível em: http://dab.saude.gov.br/CNSB/ brasil_sorridente.php

7. Morris AJ, Burke FJT. Primary and secondary dental care: how ideal is the interface? Br Dent $J$ 2001; 191(12):666-670.

8. Brasil. Ministério da Saúde (MS). Coordenação Nacional de Saúde Bucal. Brasil Sorridente. Centros de Especialidades e Laboratórios de Próteses. [acessado 2014 jun 14]. Disponível em: http://dab.saude.gov.br/CNSB/ especialidades.php

9. Conill EM. Avaliação da integralidade: conferindo sentido para os pactos na programação de metas dos sistemas municipais de saúde. Cad Saude Publica 2004; 20(5):1417-1423. 
10. Brasil. Conselho Nacional de Secretários de Saúde (CONASS). Coleção Para Entender a Gestão do SUS. Regulação em Saúde. Brasília; 2011. [acessado 2014 jul 14]. Disponível em: http://bvsms.saude.gov.br/bvs/publicacoes/para_entender_gestao_sus_v.10.pdf

11. Kornis GEM, Maia LS, Fortuna RFP. Evolução do financiamento da atenção à saúde bucal no SUS: uma análise do processo de reorganização assistencial frente aos incentivos federais. Physis 2011; 21(1):197-215.

12. Brasil. Ministério da Saúde (MS). Portaria No 2.898 de 21 de setembro de 2010. Atualiza o Anexo da Portaria No600/GM/ MS, de 23 de março de 2006. Diário Oficial da União 2010; $31 \mathrm{dez}$.

13. Brasil. Ministério da Saúde (MS). Departamento de Informática do Sistema Único de Saúde (Datasus). Index. [acessado 2014 jul 14]. Disponível em: http://www2. datasus.gov.br/Datasus/index.php

14. Instituto Brasileiro de Geografia e Estatística (IBGE). Censo demográfico 2010. [acessado 2014 jul 14]. Disponível em: http://www.ibge.gov.br/home/estatistica/ populacao/censo2010/default.shtm

15. Brasil. Ministério da Saúde (MS). Pesquisa Saúde: facilitando a apropriação do conhecimento científico na gestão de saúde. Rev. Saude Publica 2010; 44(4):975-978.

16. Brasil. Ministério da Saúde (MS). Secretaria de Atenção à Saúde, Departamento de Atenção Primária, Coordenação Nacional de Saúde Bucal. Nota Técnica. [acessado 2014 jul 14]. Disponível em: http://189.28.128.100/ dab/docs/geral/historico_cobertura_sf_nota_tecnica. pdf

17. Donabedian A. Aspects of medical care administration: specifying requirements for health care. Cambridge: Harvard University; 1973.

18. Sanchez RM, Ciconelli RM. Conceitos de acesso à saúde. Rev Panam Salud Publica 2012; 31(3):260-268.

19. Martins RC, Seijo MOS, Ferreira EF, Paiva SM, Ribeiro Sobrinho AP. Dental Students' Perceptions about the Endodontic Treatments Performed Using NiTi Rotary Instruments and Hand Stainless Steel Files. Braz Dent $J$ 2012; 23(6):729-736

20. Mello GA, Viana ALD. Uma história de conceitos na saúde pública. História, Ciências, Saúde 2012; 19(4):1219-1239.

21. Volpato LER, Scatena JH. Análise da política de saúde bucal do Município de Cuiabá, Estado de Mato Grosso, Brasil, a partir do banco de dados do Sistema de Informações Ambulatoriais do Sistema Único de Saúde (SIA-SUS). Epidemiol Serv Saúde 2006; 15(2):47-55.

22. Matos CA, Pompeu JC. Onde estão os contratos? Análise da relação entre os prestadores privados de serviços de saúde e o SUS. Cienc Saude Colet 2003; 8(2):629-643.

23. Nicoletto SCS, Cordoni Junior L, Costa NR. Consórcios intermunicipais de saúde do Paraná. Cad Saude Publica 2005, 21(1):29-38.

24. Siqueira-Batista R, Gomes AP, Albuquerque VS, Cavalcanti FOL, Cotta RMM. Educação e competências para o SUS: é possível pensar alternativas à(s) lógica(s) do capitalismo tardio? Cien Saude Colet 2013; 18(1):159170.
25. Figueiredo N, Goes PSA. Construção da atenção secundária em saúde bucal: um estudo sobre os Centros de Especialidades Odontológicas em Pernambuco, Brasil. Cad Saude Publica 2009; 25(2):259-267.

26. Junqueira SR, Araújo ME, Antunes JLF, Narvai PC. Indicadores socioeconômicos e recursos odontológicos em municípios do estado de São Paulo, Brasil, no final do século XX. Epidemiologia e Serviços de Saude 2006; 15(4):41-53.

27. Minas Gerais. Secretaria Estadual de Saúde. Políticas de Saúde. Plano Diretor de Regionalização-PDR. 20012004. [acessado 2014 jul 14]. Disponível em: https:// www.nescon.medicina.ufmg.br/biblioteca/imagem/ 3022.pdf

28. Minas Gerais. Secretaria Estadual de Saúde. Atos Normativos: Deliberação CIB-SUS No 407, de 22 de novembro de 2007. Institui a Rede Estadual e define os critérios, normas e requisitos para a implantação e credenciamento dos Centros de Especialidades Odontológicas (CEO) e dos Laboratórios Regionais de Próteses Dentárias (LRPD) em Minas Gerais. Diário Oficial do Estado de Minas Gerais 2007; 23 nov.

29. Berretta IQ, Lacerda JT, Calvo MCM. Modelo de avaliação da gestão municipal para o planejamento em saúde. Cad Saude Publica 2011; 27(11):2143-2154.

30. Goes PSA, Figueiredo N, Neves JC, Silveira FMM, Costa JFR, Pucca Júnior, GA, Rosales MS. Avaliação da atenção secundária em saúde bucal: uma investigação nos centros de especialidades do Brasil. Cad Saude Publica 2012; 28(Supl.):S81-S89.

31. Chaves SCL, Silva LMV. Atenção à saúde bucal e a descentralização da saúde no Brasil: estudo de dois casos exemplares no Estado da Bahia. Cad Saude Publica 2007; 23(5):1119-1131.

32. Bodstein R. Atenção básica na agenda da saúde. Cien Saude Colet 2002; 7(3):401-412.

33. Mendes EV. As redes de atenção à saúde. Cien Saude Colet 2010; 15(5):2297-2305.

34. Barros SG, Chaves SLA. Utilização do Sistema de informações Ambulatoriais (SIA-SUS) com instrumento para caracterização das ações de saúde bucal. Epidemiol Serv Saude 2003; 12(1):41-51.

35. Contandriopoulos AP. Avaliando a institucionalização da avaliação. Cien Saude Colet 2006; 11(3):705-711.

36. Tanaka OY, Tamaki EM. O papel da avaliação para a tomada de decisão na gestão de serviços de saúde. Cien Saude Colet 2012; 17(4):821-828.

37. Aquino R, Barreto ML. Programa de Saúde da Família: acerca da adequação do uso de seu indicador de cobertura. Cad Saude Publica 2008; 24(4):905-914.

Artigo apresentado em 19/06/2013

Aprovado em 15/07/2013

Versão final apresentada em 19/07/2013 